\title{
Adaptive Beamforming for Array Imaging of Plate Structures Using Lamb Waves
}

\author{
Marcus Engholm and Tadeusz Stepinski
}

\begin{abstract}
Lamb waves are considered a promising tool for the monitoring of plate structures. Large areas of plate structures can be monitored using active arrays employing beamforming techniques. Dispersion and multiple propagating modes are issues that need to be addressed when working with Lamb waves. Previous work has mainly focused on standard delay-and-sum (DAS) beamforming while reducing the effects of multiple modes through frequency selectivity and transducer design.

This paper presents a minimum variance distortionless response (MVDR) approach for Lamb waves using a uniform rectangular array (URA) and a single transmitter. Theoretically calculated dispersion curves are used to compensate for dispersion. The combination of the MVDR approach and the two-dimensional array improves the suppression of interfering Lamb modes.

The proposed approach is evaluated on simulated and experimental data and compared with the standard DAS beamformer. It is shown that the MVDR algorithm performs better in terms of higher resolution and better side lobe and mode suppression capabilities. Known issues of the MVDR approach, such as signal cancellation in highly correlated environments and poor robustness, are addressed using methods that have proven effective for the purpose in other fields of active imaging.
\end{abstract}

\section{INTRODUCTION}

$\mathrm{I}^{\mathrm{N}}$ $\mathrm{N}$ the field of structural health monitoring (SHM) of plate structures, two main technical approaches have emerged. The first relies on a relatively dense network of transceivers covering the whole structure, and the second, which is the subject of this paper, on active arrays, in which each array is capable of monitoring a larger area of the plate.

Plates act as waveguides for elastic waves. For infinite, traction free, thin plates (thin in the sense that the thickness is comparable to the wavelength) this results in Lamb waves. Lamb waves are advantageous because they can propagate over long distances, making them suitable for structural health monitoring (SHM) and non-destructive testing (NDT) of plate structures. As with other types of guided waves, there is more than one possible propagating mode for each frequency. Each Lamb mode is characterized by a dispersion curve relating frequency to phase velocity.

Manuscript received December 1, 2009; accepted September 2, 2010. This work was supported by SKB (the Swedish Nuclear Fuel and Waste Management Co.) in the project 1052/09.

The authors are with Uppsala University, Signals and Systems Group, Uppsala, Sweden (e-mail: marcus.engholm@gmail.com).

Digital Object Identifier 10.1109/TUFFC.2010.1745
The multiple propagating modes and the dispersion are two important issues that need to be addressed when Lamb waves are utilized for inspection or monitoring. Most of the previously presented approaches for Lamb wave imaging have focused on the standard delay-andsum (DAS) beamformer [1]-[7]. The small arrays typically employed in this type of setup offer poor suppression of unwanted, or interfering, Lamb modes when used with the DAS beamformer. The use of larger arrays could, of course, improve the suppression of interfering modes, in the same way as for signals from other angles. However, this may be impractical or too expensive. Mode selectivity has instead been achieved on the sensor level. For example, Wilcox used a circular array of electro-mechanical acoustic transducers (EMAT) to generate symmetric modes [3], and Giurugiutiu proposed tuning of the geometric shape of the array elements to achieve mode selectivity for a certain frequency range [8]. A disadvantage of such methods is that mode selectivity is generally limited to a relatively narrow frequency band [9], which poses a limitation on the bandwidth. Limited bandwidth has a negative effect on range resolution.

Dispersion reduces the spatial and temporal resolution, which is a problem for imaging applications where range is to be estimated. In an environment where there is a dominant mode, it is possible to select a frequency region where waves have relatively low dispersion and thereby reducing the problem. Wilcox used the theoretically calculated dispersion curves for the plate to transform time domain data into dispersion compensated spatial domain data over a wider frequency range for a single mode [3], [10].

Other work on Lamb wave arrays include Moulin et al. [11] who examined the beam steering properties of a small array in transmission. Fromme et al. [12] used the approach in [3] on an array of piezoelectric transducer elements designed for mode selectivity. In [13], Velichko and Wilcox proposed a data independent method to maximize the ratio between the amplitude in a certain direction and the sum of the contributions from all directions through weight vector optimization for multiple transmitter setups. For single transmitter setups this results in the wellknown beamwidth-side lobe level trade-off.

Advanced array processing methods, such as adaptive beamforming, have not been extensively used in Lamb wave applications. Adaptive beamforming has been successfully utilized in, for example, radar, sonar, and medical ultrasound applications, resulting in higher resolution and better side lobe suppression compared with the standard DAS beamformer. The difference between the DAS 


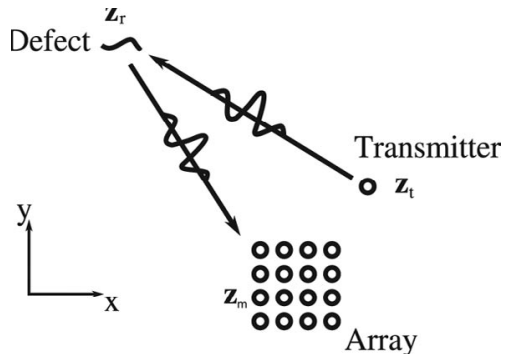

Fig. 1. Overview of the imaging problem.

beamformer, which is independent of data, and an adaptive beamformer is that the latter is optimized based on some data criterion, such as minimizing the influence of interfering signals in the received data. One of the more commonly used adaptive array processing techniques is the minimum variance distortionless response (MVDR) method. A comparison between the MVDR method and a standard beamformer for passive direction-of-arrival (DOA) estimation of Lamb waves was presented in [14]. The results showed that the MVDR approach outperforms the standard beamformer in terms of resolution and side lobe level.

In this paper, an MVDR approach for Lamb wave imaging is proposed which is capable of working in the highly correlated environment of an active setup. The basic steps of the method follow the approach used in medical ultrasound by Sasso and Cohen-Bacrie [15], Synnevåg et al. [16], and others. Here, the technique is extended to handle dispersion and 2-D arrays. The motivation for using this approach is its high resolution and its ability to adaptively suppress interfering signals from other defects and from other propagating Lamb modes. Efficient suppression of interfering modes may relax the requirements on transducer design and limitations in signal bandwidth.

The paper is organized as follows, basic Lamb wave theory and the simulation model are described first. Before explaining the theoretical steps of the proposed method, the standard DAS beamformer and the MVDR beamformer are reviewed. The theoretical presentation is followed by some results in which the proposed method is compared with the DAS beamformer using both simulated and experimental data.

\section{Problem Formulation}

This work considers the monitoring of thin plates through pulse-echo ultrasonic imaging using guided Lamb waves. The imaging covers $360^{\circ}$, in both near-field and far-field using a rectangular array as illustrated in Fig. 1. A single transmitter located at $\mathbf{z}_{t}=\left[x_{t}, y_{t}\right]^{T}$, that may be an array element or a separate transducer, insonifies the plate with a short pulse or a time-windowed sinusoid. The resulting waves are reflected back from boundaries and defects and the backscattered field is recorded by the array elements. Let $g_{m}(t)$ denote the signal received by the $m$ th array element. These signals can either be obtained simultaneously or multiplexed through repeated transmitter excitation. The objective is to estimate the power originating from each point $\mathbf{z}=[x, y]^{T}$ in the region of interest.

Two issues related to Lamb waves that must be addressed are the dispersion and the multiple modes. Furthermore, because an active setup is considered, the backscattered signals derive from the same transmit pulse and must therefore be considered as highly correlated. Henceforth, it is assumed that $g_{m}(t)$ is the analytic (complex) signal; thus, all negative frequency components are zero.

\section{TheoreticAL BACKGRound}

\section{A. Dispersion}

To perform imaging using Lamb waves over a wide frequency band, the dispersive properties of the Lamb waves must be addressed. A necessary step in both simulation and compensation of dispersion is the calculation of the theoretical dispersion characteristics of the monitored structure. For homogeneous isotropic plates, the wavenumber at angular frequency $\omega, k(\omega)$, can be calculated by solving the Rayleigh-Lamb frequency equations [9],

$$
\begin{aligned}
& \frac{\tan (q d / 2)}{\tan (p d / 2)}=-\frac{4 k^{2}(\omega) p q}{\left(q^{2}-k^{2}(\omega)\right)^{2}} \quad \text { for symmetric modes, } \\
& \frac{\tan (q d / 2)}{\tan (p d / 2)}=-\frac{\left(q^{2}-k^{2}(\omega)\right)^{2}}{4 k^{2}(\omega) p q} \quad \text { for antisymmetric modes, }
\end{aligned}
$$

where $d$ is the thickness of the plate, $p^{2}=\left(\omega / c_{\mathrm{L}}\right)^{2}-k^{2}(\omega)$, and $q^{2}=\left(\omega / c_{\mathrm{S}}\right)^{2}-k^{2}(\omega) ; c_{\mathrm{L}}$ is the longitudinal bulk wave velocity and $c_{\mathrm{S}}$ is the shear bulk wave velocity of the material.

For notational convenience, let index $n$ denote any of the possible Lamb modes propagating in the plate, for example, the symmetric $S_{0}$ mode or the antisymmetric $A_{0}$ mode. The mode-dependent wavenumber of mode $n$ is denoted $k_{n}(\omega)$.

\section{B. Simulation Model}

Consider the setup illustrated Fig. 1. To simplify the mathematical description, the model is described in the frequency domain as harmonic components. Assume that the transmitter at position $\mathbf{z}_{t}$ generates a harmonic surface point force normal to the plate, denoted $\mathcal{T}(\omega)$. This excites a cylindrical wave consisting of one or more Lamb modes in the plate. The excitation of mode $n$ is modeled by the transfer function $H_{n}(\omega)$, that relates the out-ofplane point force to the out-of-plane displacement of the mode. The excitation depends on the so-called mode shape of the Lamb mode and its wavenumber. The mode shape is the vertical displacement pattern of each mode and it is determined by the plate's thickness and material proper- 


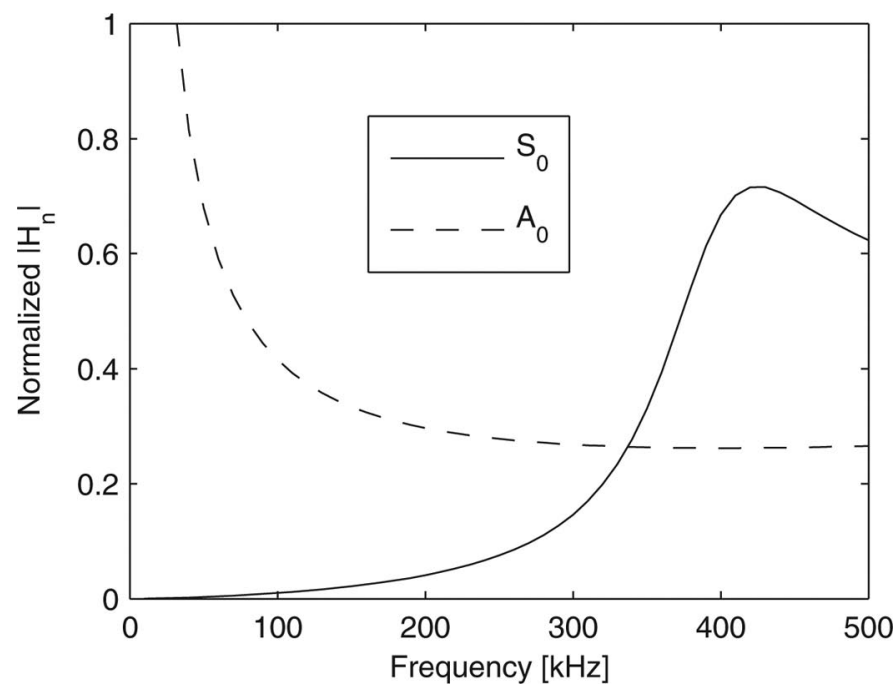

Fig. 2. Normalized excitability of modes $S_{0}$ and $A_{0}$.

ties. The equations describing the excitability of Lamb modes for a particular frequency of a point source excitation has been derived in many publications. Because the complete expressions for $H_{n}(\omega)$ are rather lengthy, the interested reader may consult, for example, [17] for details. Fig. 2 shows the excitability in terms of normalized amplitudes of $H_{n}(\omega)$ for the $S_{0}$ and $A_{0}$ modes for the plate that was used in the evaluation.

Assume a plate with $R$ reflectors that reflect incident waves equally in all directions. Further assume that there are no multiple reflections between the reflectors. Let $G_{m}(\omega)$ denote the signal received by array element $m$ with angular frequency $\omega$. Each reflector results in a signal that is a superposition of the existing modes and the corresponding phase-shifts caused by the dispersion in the propagation path from the transmitter to the reflector $r$, and back to the receiving element $m$. Assuming that the measured signal from each array element represents exactly the out-of-plane displacement at its positions, the received signals are simply the sum of the contributions from all existing modes and reflectors:

$$
G_{m}(\omega)=\sum_{r=1}^{R} \sum_{n} \frac{1}{\sqrt{z_{r}}} R_{r} H_{n}(\omega) \mathcal{T}(\omega) e^{-j k_{n}(\omega) z_{r}}
$$

where $z_{r}=\left\|\mathbf{z}_{r}-\mathbf{z}_{m}\right\|+\left\|\mathbf{z}_{r}-\mathbf{z}_{y}\right\|$, and $\|\cdot\|$ denotes the Euclidean norm. The position of the reflector is denoted $\mathbf{z}_{r}, \mathbf{z}_{m}$ is the position of the receiver, and $\mathbf{z}_{t}$ is the position of the transmitter, as illustrated in Fig. 1. The reflection coefficient, $R_{r}$, is assumed to be frequency-independent and equal for all modes. In practical applications it must be expected that the reflection coefficient is both modeand frequency-dependent. Mode conversion may also occur upon reflection, where parts of the energy of the incident mode are reflected as another mode. It is also assumed that the displacement fields from the point-like transmitters can be approximated by the divergence factor $1 / \sqrt{z_{r}}$ and the exponential $e^{-j k_{n}(\omega) z_{r}}$.

\section{Minimum Variance Distortionless Response}

To place the proposed approach into context, this section gives a brief review of the DAS and MVDR methods for narrowband far-field beamforming for 2-D arrays. The delay-and-sum (DAS) beamformer is the most commonly used technique for beamforming. Its main advantage is robustness, which comes at the cost of poor performance compared with more advanced methods. The DAS beamformer uses predetermined weights on the input signals and is therefore independent of the received data.

The output from a narrowband beamformer is

$$
y(t)=\mathbf{w}^{\mathrm{H}} \mathbf{x}(t)
$$

where ${ }^{\mathrm{H}}$ is the conjugate transpose, $\mathbf{w}$ is the complex valued column weight vector, and $\mathbf{x}(t)$ is a column vector containing the narrowband ${ }^{1}$ input signals from each array element at time $t$.

Let $k_{x}=k \cos (\theta)$ and $k_{y}=k \sin (\theta)$ denote the wavenumber components ${ }^{2}$, where $\theta$ is the incidence angle and $k$ the wavenumber. For the narrowband case, the DAS weight vector is simply set to the steering vector $\mathbf{a}\left(k_{x}, k_{y}\right) / M$, where $M$ is the number of array elements. It is also common to apply some window function to give different weights to the elements (apodization) for the purpose of lowering the amplitude of the side lobes in the beampattern. This comes at the cost of reduced resolution.

The steering vector for a uniform rectangular array (URA) can be formed by stacking rows of ULA steering vectors [18]. Consider a URA with $M_{\mathrm{c}}$ columns, and $M_{\mathrm{r}}$ rows, with a total number of $M$ elements. Let the steering vector for the $m_{\mathrm{r}}$ row be

$\mathbf{a}_{m_{\mathrm{r}}}\left(k_{x}, k_{y}\right)=\left[\begin{array}{llll}e^{j m_{\mathrm{r}} k_{y} d} & e^{j\left(k_{x} d+m_{\mathrm{r}} k_{y} d\right)} & \cdots & e^{j\left(\left(M_{\mathrm{c}}-1\right) k_{x} d+m_{\mathrm{r}} k_{y} d\right)}\end{array}\right]^{T}$,

where $d$ is the element spacing. The stacked steering vector takes the form

$$
\mathbf{a}\left(k_{x}, k_{y}\right)=\left[\begin{array}{c}
\mathbf{a}_{0}\left(k_{x}, k_{y}\right) \\
\vdots \\
\mathbf{a}_{M_{\mathrm{r}}}\left(k_{x}, k_{y}\right)
\end{array}\right] .
$$

The unwindowed output of the standard beamformer is

$$
y_{\mathrm{BF}}\left(t, k_{x}, k_{y}\right)=\frac{1}{M} \mathbf{a}^{\mathrm{H}}\left(k_{x}, k_{y}\right) \mathbf{g}(t) .
$$

Without any constraints, the DAS beamformer is simply a filter with unit gain for signals having wavenumber $\left(k_{x}, k_{y}\right)$.

The MVDR method, also known as Capon's method, was originally proposed for frequency-wavenumber estimation of seismic waves [19]. The MVDR method sets a

\footnotetext{
${ }^{1} \mathrm{~A}$ narrowband signal has a complex envelope that can be considered constant during its propagation over the array.

${ }^{2}$ Note that $k_{x}$ and $k_{y}$ represent the direction of arrival. This differs from the wavenumber vector, which describes the direction of propagation, by a minus sign.
} 
data-dependent weight vector which minimizes the influence of interfering signals while passing the desired signal undistorted. Common to many other advanced array processing methods, the standard MVDR approach assumes narrowband signals and is unable to handle correlated sources. Besides requiring that the gain for signals with wavenumber $\left(k_{x}, k_{y}\right)$ is 1 , i.e., $\mathbf{w}^{\mathrm{H}} \mathbf{a}\left(k_{x}, k_{y}\right)=1$, it also requires the filter to minimize the output power from the array, $\min _{\mathbf{w}} \mathbf{w}^{\mathrm{H}} \mathbf{R} \mathbf{w}[20]$. $\mathbf{R}$ is the covariance matrix, $\mathbf{R}=$ $\mathrm{E}\left\{\mathbf{g}(t) \mathbf{g}^{\mathrm{H}}(t)\right\}$, where $\mathrm{E}$ denotes the expected value. Thus, the covariance matrix consists of the covariances between the signals received by the different array elements, and is in practice replaced by the sample covariance matrix

$$
\hat{\mathbf{R}}=\frac{1}{N} \sum_{t=1}^{N} \mathbf{g}(t) \mathbf{g}^{\mathrm{H}}(t) .
$$

This means that the algorithm produces a weight vector that minimizes the influence of the interferers. The solution to this optimization problem is simply [20]

$$
\mathbf{w}=\frac{\hat{\mathbf{R}}^{-1} \mathbf{a}\left(k_{x}, k_{y}\right)}{\mathbf{a}^{\mathrm{H}}\left(k_{x}, k_{y}\right) \hat{\mathbf{R}}^{-1} \mathbf{a}\left(k_{x}, k_{y}\right)} .
$$

The estimated power of the signal with wavenumber $\left(k_{x}, k_{y}\right)$ is

$$
P_{\mathrm{MVDR}}\left(k_{x}, k_{y}\right)=\frac{1}{\mathbf{a}^{\mathrm{H}}\left(k_{x}, k_{y}\right) \hat{\mathbf{R}}^{-1} \mathbf{a}\left(k_{x}, k_{y}\right)} .
$$

A major disadvantage of the MVDR algorithm is that it is sensitive to errors in the steering vector, where even small errors can lead to underestimation of the desired signal. Another issue that needs to be addressed is that the MVDR method is incapable of handling highly correlated sources, which may lead to cancellation of the signal of interest. For the steering vector error, this leads to underestimation of the signal amplitude. Finally, broadband signals cannot be modeled using the narrowband steering vector in (6). The following sections address these issues and propose suitable solutions for MVDR imaging of Lamb waves.

\section{Methods}

There are some fundamental differences between active near-field imaging applications and traditional farfield passive sensing for which the MVDR algorithm was originally proposed. This section will describe the steps required to employ the MVDR approach for Lamb wave imaging.

\section{A. Focusing}

In previous work on medical ultrasound imaging [15], [16], the so-called steered covariance matrix approach [21] has been used for near-field focusing. Applying delays to focus the data to a particular point enables the estimation of a broadband covariance matrix where the steering vector for all frequency components will be equal to the unit vector, 1, at that point. This enables direct application of the MVDR method to calculate a weight vector for the steered data. However, because the frequency components of Lamb waves propagate at different phasevelocities, simply applying time-delays will not align the signals properly. By performing the focusing in frequency domain, each frequency component can be phase-shifted using the dispersion characteristics of the plate, thereby compensating for the propagation over the transmitterreflector-receiver path. This is performed for a particular mode $n$ as [10]

$$
h_{m, n}(\mathbf{z})=\sqrt{z_{d}} \sum_{\omega} G_{m}(\omega) e^{j k_{n}(\omega) z_{d}}
$$

where $z_{d}=\left\|\mathbf{z}-\mathbf{z}_{m}\right\|+\left\|\mathbf{z}-\mathbf{z}_{t}\right\|$. The first factor compensates for the divergence in the plate. The focused signals are arranged in the array

$$
\mathbf{h}_{n}(\mathbf{z})=\left[\begin{array}{llll}
h_{1, n}(\mathbf{z}) & h_{2, n}(\mathbf{z}) & \cdots & h_{M, n}(\mathbf{z})
\end{array}\right]^{T} .
$$

This operation is performed at each point in a grid covering the monitored region of the plate. Because it is assumed that $g_{m}(t)$ is the analytic signal, only frequency components below the Nyquist frequency are nonzero.

\section{B. Spatial Smoothing}

Recall that the backscattered signals in an active setup are likely highly correlated. Thus, the most significant limitation of the MVDR algorithm for array imaging applications is its deficiency in handling correlated signals. As mentioned in Section III-C, this may result in signal cancellation causing underestimation of the amplitude of the reflected signals. Several different approaches have been proposed to reduce signal cancellation [22], [23]. In the medical ultrasound field [15], [16] this problem has been mitigated through so-called spatial smoothing [22] of linear arrays. This approach can be directly extended to rectangular arrays.

For a single transmitter, each point in focus, $\mathbf{z}$, will result in a vector $\mathbf{h}_{n}(\mathbf{z})$ that can be used to form a focused sample covariance matrix with rank one, $\hat{\mathbf{R}}_{n}(\mathbf{z})=\mathbf{h}_{n}(\mathbf{z}) \mathbf{h}_{n}^{\mathrm{H}}(\mathbf{z})$.

A rank one covariance matrix can, of course, only represent a single scatterer. To capture the statistics in a multiscatterer and multimode environment, the rank of the covariance matrix must be increased using multiple snapshots. The sample covariance matrix in (8) is formed by temporal averaging over several samples, or snapshots, and can thereby acquire a sufficient rank. For the focused covariance matrix, this could be achieved through spatial averaging, which is performed by focusing and forming covariance matrices over several points close to the point 


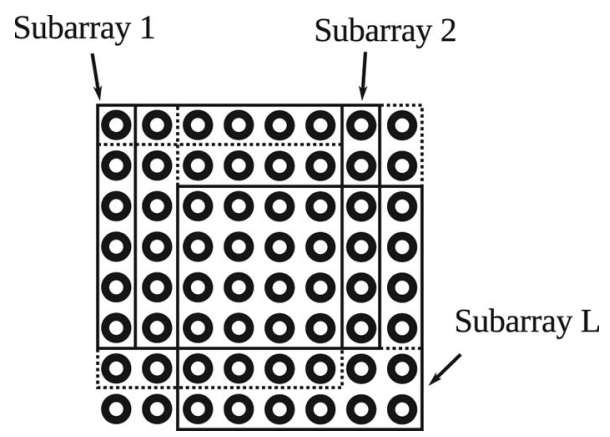

Fig. 3. Spatial smoothing. The array is divided into $L$ overlapping subarrays.

of interest. The covariance matrices are then averaged, resulting in a spatially averaged covariance matrix. However, because the broadband signals are spatially localized, averaging over adjacent points may not improve the estimate. Furthermore, spatial or temporal averaging of this kind does not decorrelate the signals.

The spatial smoothing approach addresses both of these problems; it decorrelates signals and increases the number of snapshots. Spatial smoothing is performed by dividing the array into $L$ rectangular subarrays, see Fig. 3, each consisting of $M^{\prime}$ elements. The covariance matrices calculated for each of the subarrays are then averaged. The approach requires the subarrays to have identical shape, which poses a limitation on the shape of the array. This motivates using a rectangular array instead of, for example, a circular array. The disadvantage with the approach is that the effective aperture size is reduced to that of the subarrays.

In [24], an optimal subarray size of a uniform linear array for two closely spaced narrowband coherent sources was derived. It was shown that an optimal size of subarrays for a uniform linear array is 0.6 (number of array elements +1$)$. However, it should be expected that backscattered signals from two defects are not perfectly coherent because they are never identical. This could motivate a slightly larger subarray size for measurement data than the optimal size for ideal coherent signals.

The focused signals from element $m^{\prime}$ of subarray $l$ are denoted $h_{l, m^{\prime}, n}(t)$. The dispersion compensated and focused spatial signal of subarray $l$ is

$$
\mathbf{h}_{l, n}(\mathbf{z})=\left[\begin{array}{llll}
h_{l, 1, n}(\mathbf{z}) & h_{l, 2, n}(\mathbf{z}) & \cdots & h_{l, M^{\prime}, n}(\mathbf{z})
\end{array}\right]^{T} .
$$

Hence, the sample covariance matrix of subarray $l$ at point $\mathbf{z}$ is given by

$$
\hat{\mathbf{R}}_{l, n}(\mathbf{z})=\mathbf{h}_{l, n}(\mathbf{z}) \mathbf{h}_{l, n}^{\mathrm{H}}(\mathbf{z}) .
$$

Finally, the smoothed covariance matrix is found by averaging over the subarray covariance matrices

$$
\hat{\mathbf{R}}_{s, n}(\mathbf{z})=\frac{1}{L} \sum_{l=1}^{L} \hat{\mathbf{R}}_{l, n}(\mathbf{z}) .
$$

The decorrelating effect from the spatial smoothing can be further improved by also including the backward covariance matrix estimates through so-called forwardbackward averaging [18]. The backward covariance matrix is given by

$$
\hat{\mathbf{R}}_{s, n, \mathrm{~B}}(\mathbf{z})=\mathbf{J} \hat{\mathbf{R}}_{s, n}^{\mathrm{H}}(\mathbf{z}) \mathbf{J},
$$

where $\mathbf{J}$ is the exchange matrix, which is a square matrix with ones on the counter-diagonal and all other elements zero. The forward-backward spatially smoothed covariance matrix is the average of the forward and backward estimates,

$$
\hat{\mathbf{R}}_{s, n, \mathrm{FB}}(\mathbf{z})=\frac{1}{2}\left(\hat{\mathbf{R}}_{s, n}(\mathbf{z})+\hat{\mathbf{R}}_{s, n, \mathrm{~B}}(\mathbf{z})\right)
$$

After these steps, the power of a particular mode in point $\mathbf{z}$ can now be estimated using the MVDR algorithm. Because the covariance matrix is already focused, the steering vector is simply the unit vector, $\mathbf{1}$. This makes the MVDR-filtered output for subarray $l$

$$
y_{l, n}(\mathbf{z})=\mathbf{w}^{\mathrm{H}} \mathbf{h}_{l, n}(\mathbf{z})=\frac{\mathbf{1}^{T} \hat{\mathbf{R}}_{s, n, \mathrm{FB}}^{-1} \mathbf{h}_{l, n}(\mathbf{z})}{\mathbf{1}^{T} \hat{\mathbf{R}}_{s, n, \mathrm{FB}}^{-1}(\mathbf{z}) \mathbf{1}} .
$$

The outputs from the subarrays are then averaged

$$
y_{n}(\mathbf{z})=\mathbf{w}^{\mathrm{H}}\left(\frac{1}{L} \sum_{l=1}^{L} \mathbf{h}_{l, n}(\mathbf{z})\right),
$$

and the MVDR power estimate is calculated as

$$
\hat{P}_{\mathrm{MVDR}, n}(\mathbf{z})=\left|y_{n}(\mathbf{z})\right|^{2} .
$$

Because the DAS approach does not require spatial smoothing, it can utilize the full array. The vector $\mathbf{h}_{n}(\mathbf{z})$ is already focused, making the unwindowed DAS power estimate simply

$$
\hat{P}_{\mathrm{DAS}, n}(\mathbf{z})=\left|\mathbf{1}^{T} \mathbf{h}_{n}(\mathbf{z})\right|^{2} .
$$

Apodization can be applied by replacing the unit vector with a suitable window function.

Spatial smoothing allows the MVDR algorithm to work in the coherent signal environment of active imaging by reducing signal cancellation. Increasing the number of subarrays reduces the effects of signal cancellation, at the cost of lower resolution and interference suppression. The spatially smoothed covariance matrix has at most rank $L$. In the examples used in this work $L$ is less than the number of array elements in each subarray $M^{\prime}$, which leads to a non-invertible covariance matrix. The next section explains how the issue of invertibility can be handled and how the problem with underestimation of the signal amplitude caused by steering vector errors can be further reduced. 


\section{Robustness}

The MVDR algorithm is very sensitive to errors in the steering vector (6). A mismatch between the steering vector and the signal of interest may lead to an underestimation of the amplitude of the signal. Steering vector errors can be caused by uncertainties in element position, physical differences between array elements, or errors in the estimated dispersion characteristics of the plate. In practical implementations, some kind of regularization is often required. The simplest and most common method is diagonal loading. This is performed by adding a diagonal matrix $\alpha \mathbf{I}$ to the sample covariance matrix,

$$
\hat{\mathbf{R}}_{\text {load }}=\hat{\mathbf{R}}+\alpha \mathbf{I}
$$

where $\alpha>0$. Selecting a suitable $\alpha$ is a user-selected design parameter. There are methods for setting an optimal $\alpha$ based on the uncertainties of the steering vector [25]. A straightforward way to set $\alpha$ is to make it proportional to the average power of the received signals, which can be estimated through the trace of the covariance matrix [16]

$$
\alpha=\frac{1}{\varepsilon M^{\prime}} \operatorname{tr}\{\hat{\mathbf{R}}\}
$$

where $\varepsilon$ scales the amount of loading and $M^{\prime}$ is the number of elements in the subarrays. The diagonal loading leads to the same result as adding white noise to the signal does; it reduces the adaptivity of the MVDR algorithm and thereby also the effects of steering vector errors. To some extent, it also reduces underestimation of the amplitude caused by weakly correlated sources. Furthermore, a sufficiently large amount of diagonal loading ensures that the covariance matrix is invertible. By increasing the diagonal loading, the MVDR estimate approaches the DAS estimate.

\section{The Algorithm}

The previous sections explained in detail the proposed method. The whole procedure can be summarized as the following steps performed for each point $\mathbf{z}$ in the region of interest and for a particular Lamb mode $n$

1) Compensate for dispersion and propagation using the dispersion curve of mode $n$ for point $\mathbf{z}(11)$.

2) Form the focused array for point $\mathbf{z}(12)$.

3) Calculate covariance matrices and perform spatial smoothing (13) and (14).

4) Apply diagonal loading (22).

5) Apply the MVDR filter to calculate the estimated power (18)-(20).

The dispersion-compensated DAS approach only requires steps 1 and 2. The DAS power estimate is then given by (21).

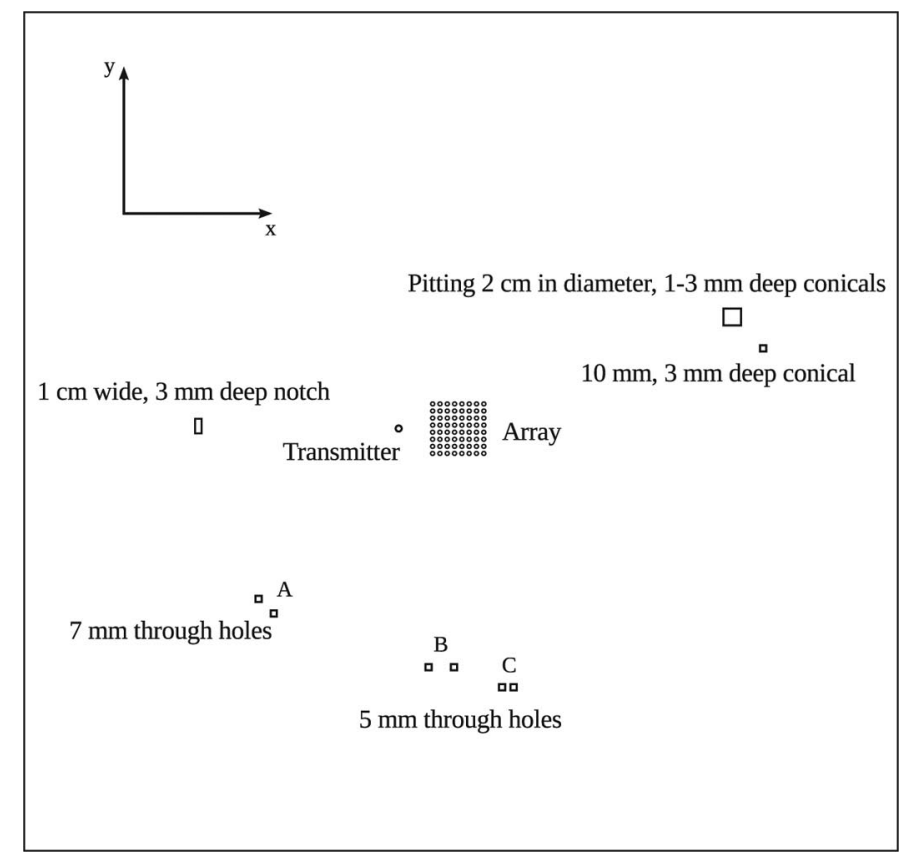

Fig. 4. Layout of the inspected aluminum plate. The labels A, B, and C identify three pairs of closely spaced holes.

\section{Results}

The proposed method was evaluated on both simulated and experimental data and compared with the DAS beamformer. The object used for the evaluation was a 6-mm-thick, $750 \times 750 \mathrm{~mm}$ aluminum plate (6082-T6) with artificial defects. The artificial defects included pairs of drilled through holes, a 1-cm-wide notch, and some artificial pits with depths 1 to $3 \mathrm{~mm}$. Because of difficulties in creating realistic simulation models for the notch and the pits, only the circular holes were used in the simulation model. The layout of the defects and the positions of the array and the transmitter are shown in Fig. 4. The pairs of holes are labeled A, B, and C. Pair A consists of two holes, $7 \mathrm{~mm}$ in diameter, $28 \mathrm{~mm}$ apart, whereas pairs $\mathrm{B}$ and $\mathrm{C}$ consist of two 5-mm holes each, located 21 and $10 \mathrm{~mm}$ apart, respectively.

A single stand-alone transducer was used for the excitation and the back-scattered signals were received by an $8 \times 8$ element rectangular array. Small pinducers from Valpey Fisher Corp. (Hopkinton, MA) were used both as receivers and transmitter. The pinducers had a diameter of $1.5 \mathrm{~mm}$ and a resonance frequency of $1.1 \mathrm{MHz}$, which was well above the frequency range used in the experiments. The pinducers were coupled to the plates through a thin layer of oil, which limited the detection and generation to out-of-plane displacement. Two different element spacings in the array were evaluated, 3.5 and $7 \mathrm{~mm}$, labeled Array 1 and 2, respectively. Spatial smoothing was applied on all results for the MVDR approach using 9 overlapping $6 \times$ 6 subarrays, thereby making the effective array size $6 \times$ 6. Following the rule derived in [24], the optimal subarray size for each side of the rectangular array would be $0.6(8$ $+1)=0.54$, which shows that a $6 \times 6$ rectangular subar- 
ray was a reasonable size. The DAS approach did not benefit from spatial smoothing, hence the full array was used for the DAS results. The signal focusing was performed using frequencies between 50 and $450 \mathrm{kHz}$.

To experimentally determine the amplitude of the transmitted Lamb modes and to validate the theoretically determined dispersion curves, a single transmitter/receiver pair was used to perform measurements allowing the calculation of a spatio-temporal FFT [26]. In this experiment, the transmitting transducer was excited by a single square pulse, $1 \mu \mathrm{s}$ long with amplitude $16 \mathrm{~V}$. The receiving transducer was shifted along a $75-\mathrm{mm}$ line in 1-mm intervals, resulting in 75 signals. The spatio-temporal FFT resulted in a multi-mode signal that can be seen in the frequency-wavenumber power spectrum in Fig. 5. From the figure, it can be seen that the received $S_{0}$ mode had a higher amplitude than the $A_{0}$ mode around $350 \mathrm{kHz}$. The longitudinal and shear wave velocities for the aluminum plate were unknown. Various velocities typical for aluminum alloys were used for calculating theoretical dispersion curves that were compared with the experimentally determined dispersion curves. A good match for the $A_{0}$ mode was achieved when setting the longitudinal wave velocity to $6198 \mathrm{~m} / \mathrm{s}$ and the shear wave velocity to $3158 \mathrm{~m} / \mathrm{s}$. The resulting theoretically determined dispersion curve is shown in Fig. 5.

Ideally, all received modes should be processed and used as a basis for the evaluation of the structure. Because of the shorter wavelength of the $A_{0}$ mode compared with the $S_{0}$ mode, and poor agreement between simulated and measured dispersion characteristics for the $S_{0}$ mode, only results from focusing using the $A_{0}$ mode dispersion characteristics are presented here. For the frequency range used in the experiments, the $A_{0}$ mode had wavelengths down to $7 \mathrm{~mm}$. To avoid spatial aliasing, this required an array element spacing of a maximum of $3.5 \mathrm{~mm}$, which was satisfied by Array 1. Using Array 2, with wider element spacing and the same number of elements as Array 1, improved resolution but could lead to aliasing effects.

Neither the simulated or experimental results benefited from spatial averaging (see Section IV-B). All results from the MVDR approach were obtained using diagonal loading according to (22).

\section{A. Simulation Results}

Two Lamb modes were included in the simulations, the $S_{0}$ and the $A_{0}$ mode. The simulations assumed reflectors with real frequency independent reflection coefficients, no multiple scattering and no mode conversion. The reflection coefficients were set to be proportional to the diameter of the holes and equal for both modes. The amplitude of the edge reflections were assumed to be 10 times the amplitude of the smallest defects, holes B and C. The notch and pits were not included in the simulation. Because the transducers were coupled to the plate using oil, the transducer-plate interaction was modeled assuming outof-plane components only. The transfer functions used for

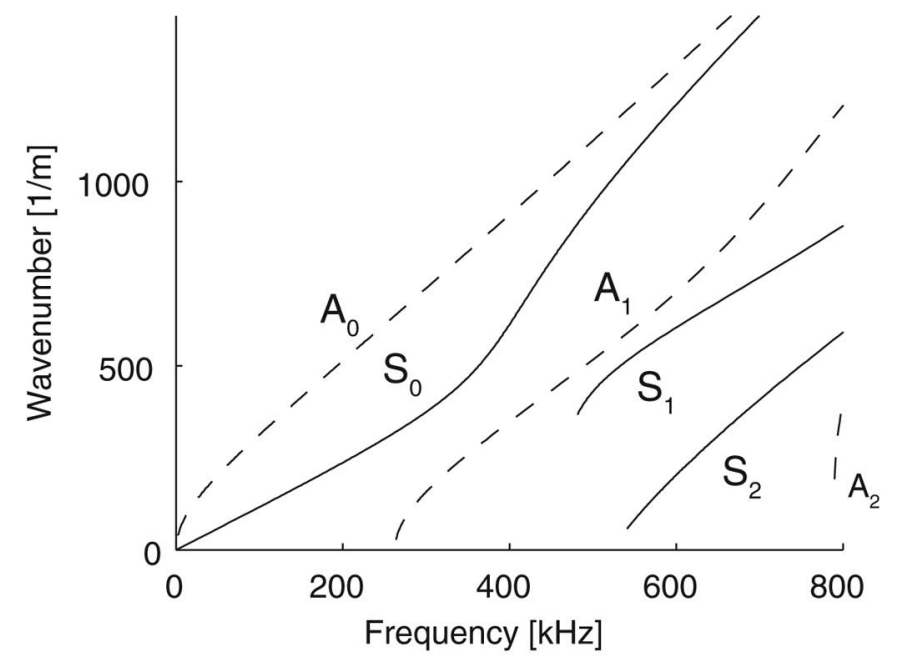

(a)

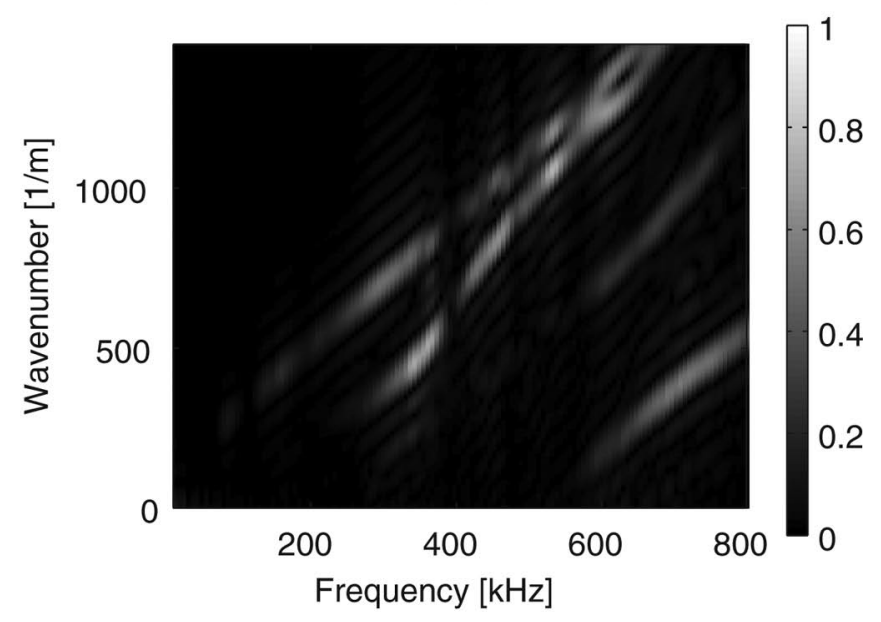

(b)

Fig. 5. Theoretically (a) and experimentally (b) determined dispersion curves for the 6 -mm aluminum plate.

this purpose were calculated using the model described in Section III-B. The input signal was a $300-\mathrm{kHz}$, single-cycle sinusoid, bandpass filtered to a bandwidth of $260 \mathrm{kHz}$. White Gaussian noise was added to the simulated received signals, giving an SNR of $25 \mathrm{~dB}$.

The theoretical level of relative excitation between the $S_{0}$ and $A_{0}$ modes, expressed as a normalized $\left|H_{n}(\omega)\right|$, is shown in Fig. 2. It can be seen that the $A_{0}$ mode has a higher excitability than the $S_{0}$ mode around $300 \mathrm{kHz}$. Because this transfer function operates on the input signal, the slope of the curve shifts the center frequency of resulting $A_{0}$ mode from 300 to $280 \mathrm{kHz}$, maintaining approximately the same bandwidth. The wavelength of the $A_{0}$ mode is $10.5 \mathrm{~mm}$ at $280 \mathrm{kHz}$.

Fig. 6 shows the result from the MVDR and the DAS on signals obtained through the simulation of Array 1 . Reflections from the hole pairs A, B, and C can be seen in the lower part of the images. The true hole positions are marked with white crosses. In some images, the defects are indicated by arrows to improve visibility. The results from Array 2 are shown in Fig. 7. 


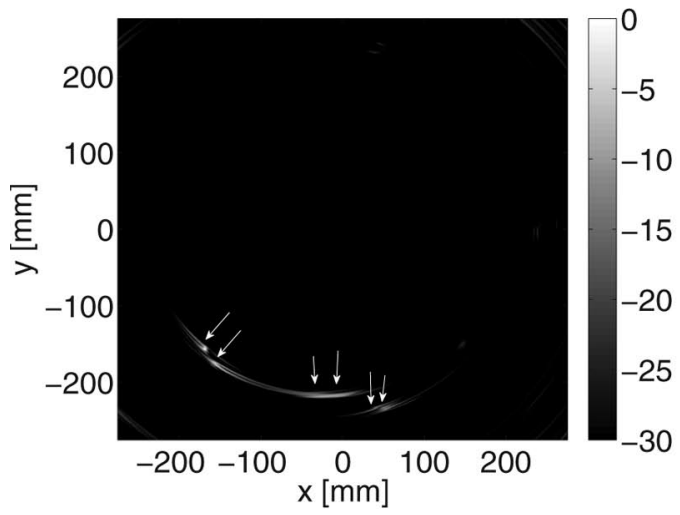

(a)

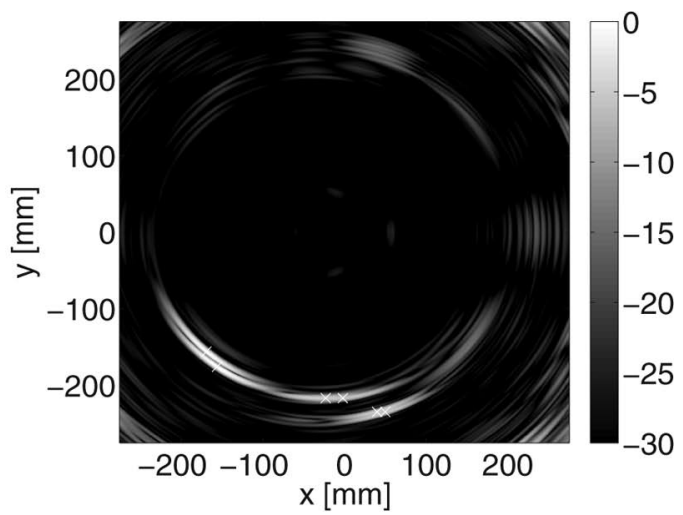

(b)

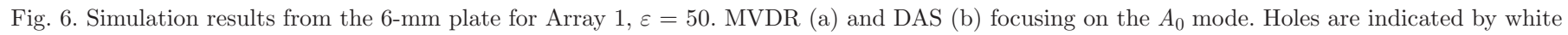
crosses or arrows. Note that the arrows do not point at the exact position of the defect. Log scale cut at $-30 \mathrm{~dB}$.

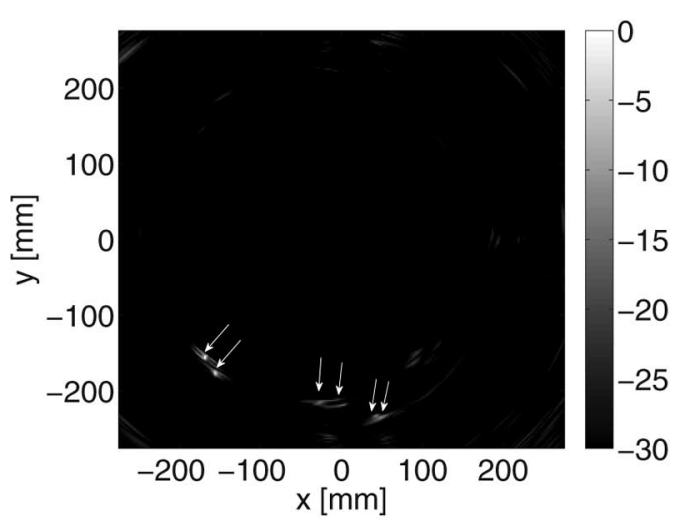

(a)

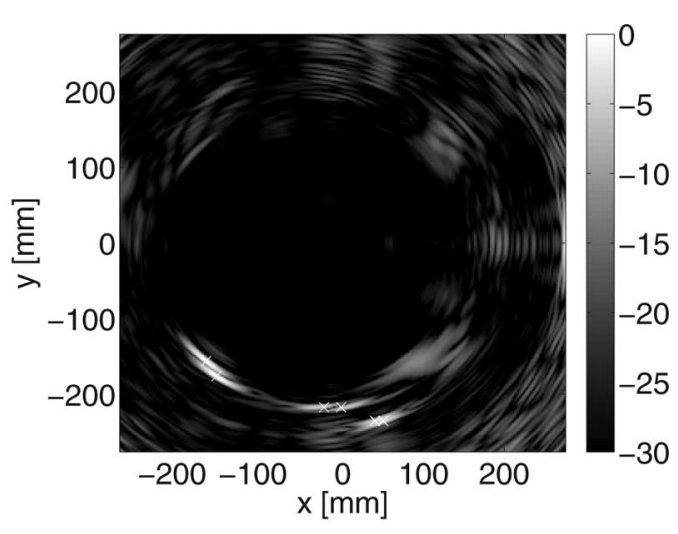

(b)

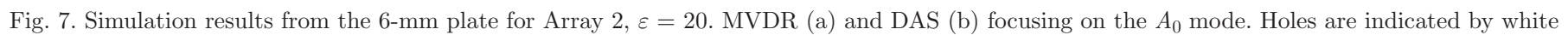
crosses or arrows. Note that the arrows do not point at the exact position of the defect. Log scale cut at $-30 \mathrm{~dB}$.

Fig. 8 shows the angle and range resolvability of the pair of holes A obtained for the results presented in Fig. 7. The projections were created by taking the maximum values in range and angle, respectively, in the vicinity of the defects. Note that the transmitter position outside the array in conjunction with the holes locations in the near field of the array, leads to elliptical beams, where the transmitter and the center of the array are in the focus points of the ellipses. This makes angle and range resolution somewhat distorted when plotting range and angle from the center of the array. The projections were therefore created using a point between the center of the array and the transmitter as a reference for range and angle.

\section{B. Experimental Results}

Because of the limited number of available transducers and limitations of the electronics only an 8-element uniform linear array was used simultaneously for the reception. The linear receiving array used in all experiments consisted of 8 pinducers with an inter-element distance of $7 \mathrm{~mm}$. The input signal was generated by an HP8116 function generator (Agilent Technologies, Santa Clara, CA) and was a single square pulse, $1 \mu$ s long with amplitude $16 \mathrm{~V}$. The array pinducers were connected to an Agilent Infiniium oscilloscope through a custom built multiplexing box followed by an AD8335 amplifier from Analog Devices (Norwood, MA). The sampling rate of the oscilloscope was set to $25 \mathrm{MHz}$. Because of the limited resolution of the oscilloscope ( 8 bits), the received edge reflections had to be saturated to get sufficient resolution of the weaker defect reflections. Increasing the dynamic range and using a 12-bit analog-to-digital converter, common in ultrasonic systems, would allow resolution of the defects without saturating the edge reflections for this particular setup. The received signal from each element was averaged 16 times.

To form the $8 \times 8$ rectangular array with $7 \mathrm{~mm}$ element spacing (Array 2) the measurements were repeated with the linear receiving array shifted at 8 different positions. Array 1 with $3.5 \mathrm{~mm}$ element spacing was formed by taking measurements at $2 \times 8$ different positions using 4 elements of the receiving array. The direct signal from the transmitter to the array was removed before processing the signals.

A comparison between the DAS and the MVDR approaches using Array 1 and 2 can be seen in Figs. 9 and 10 , respectively. The $\log$ scale is cut at $-12 \mathrm{~dB}$ compared 

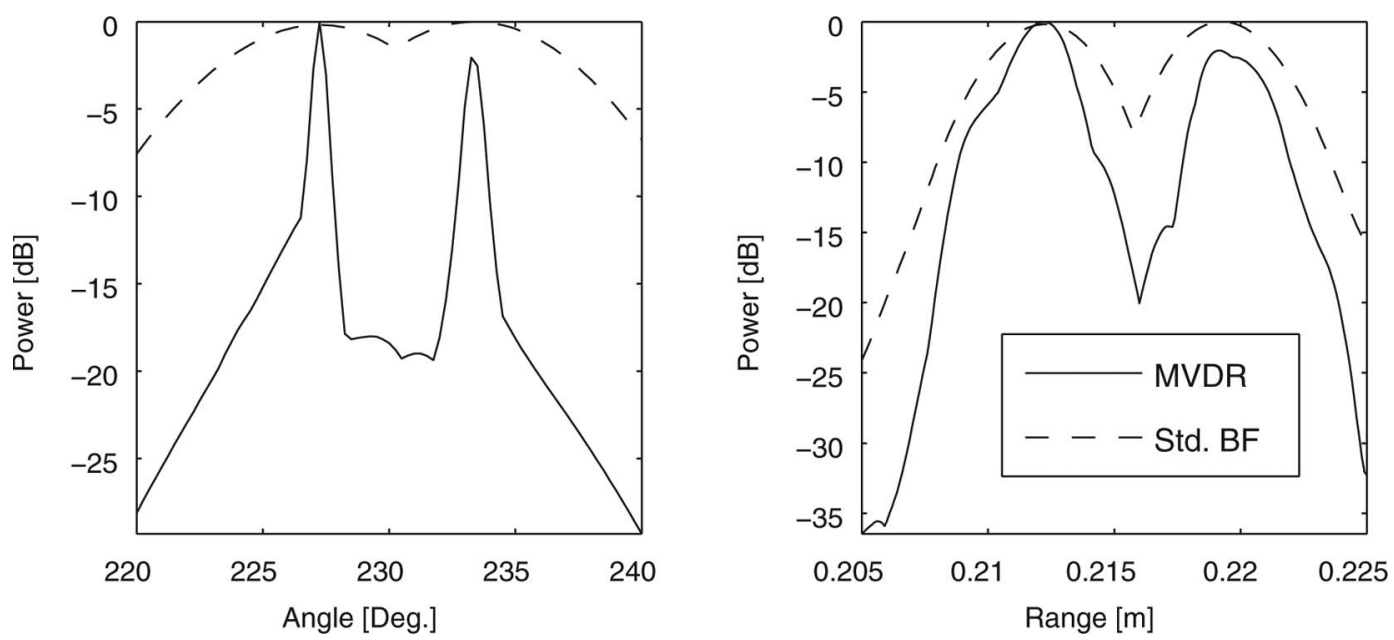

Fig. 8. Angle and range resolvability of hole pair A using the MVDR and DAS on simulated data from Array 2 on the 6-mm plate.

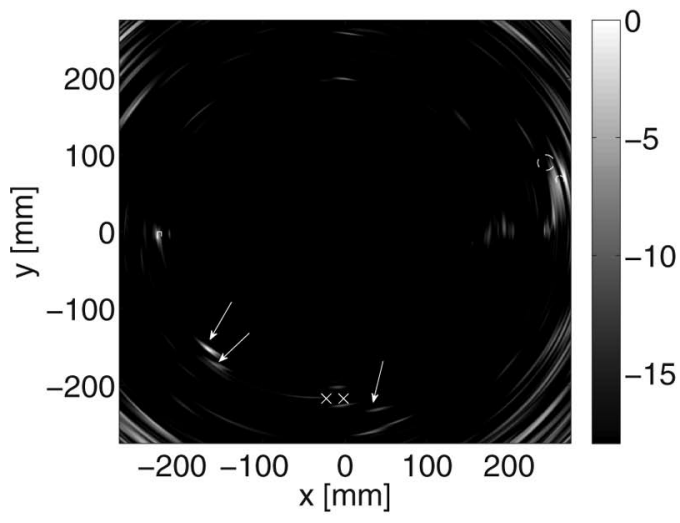

(a)

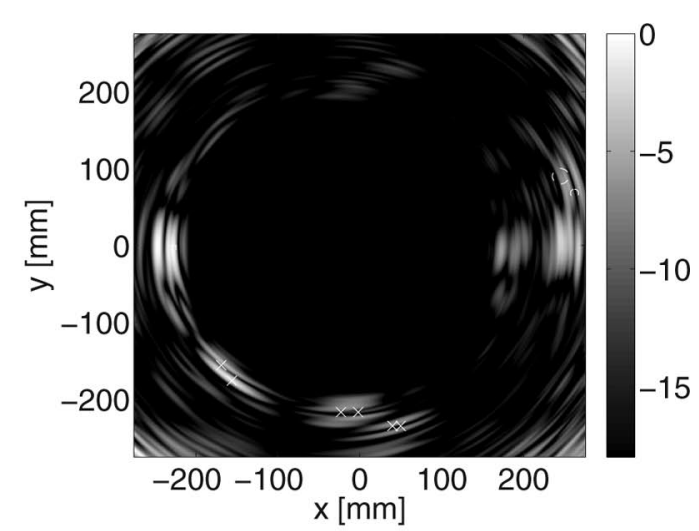

(b)

Fig. 9. Measurement results from the 6-mm plate for Array 1, $\varepsilon=50$. MVDR (a) and DAS (b) focusing on the $A_{0}$ mode. Defects are indicated by white crosses or arrows (holes, lower part of the image) and dashed lines (pittings and notch, at $0^{\circ}$ and $180^{\circ}$, respectively). Note that the arrows do not point at the exact position of the defect. Log scale cut at $-18 \mathrm{~dB}$.
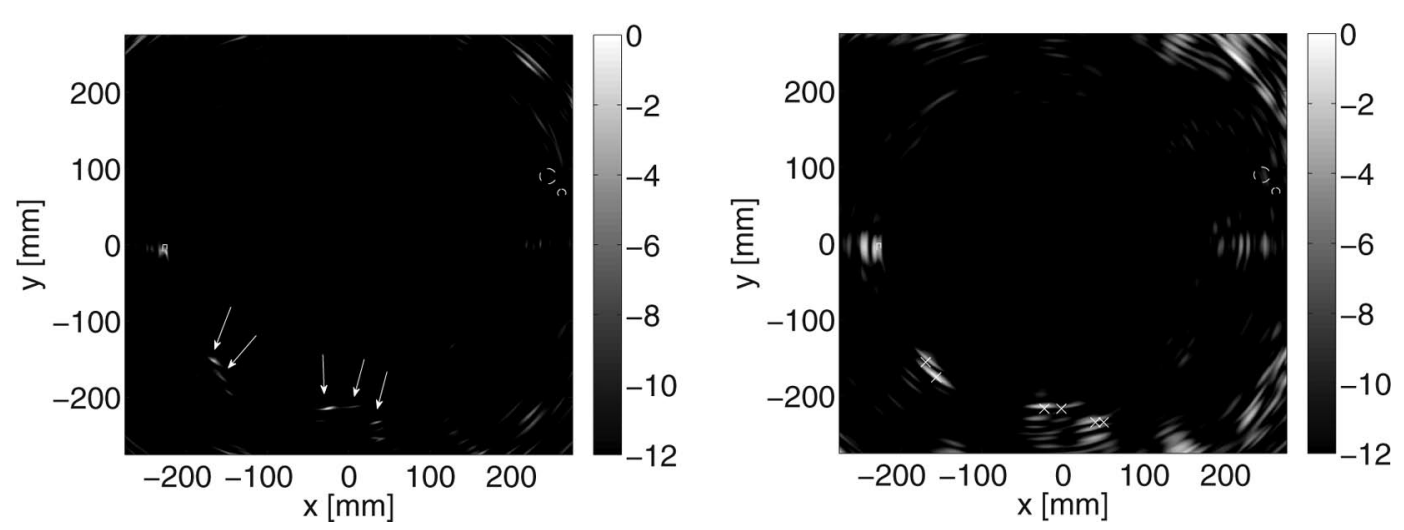

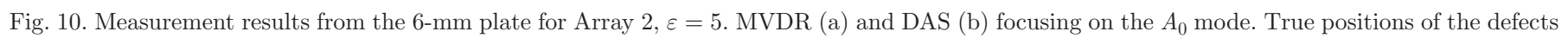

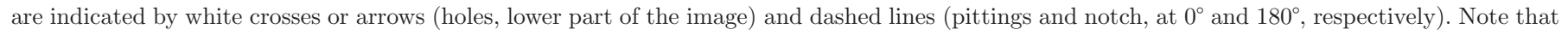
the arrows do not point at the exact position of the defect. Log scale cut at $-12 \mathrm{~dB}$.

with $-18 \mathrm{~dB}$ for Array 1 because of poor SNR. The holes are well pronounced in the lower part of the images where their true positions are marked with white crosses, or where necessary, arrows. The pit and the notch are seen at 0 and $180^{\circ}$, respectively.
Similarly to the simulated results in Fig. 8, Fig. 11 shows the angle and range resolvability of hole pair A obtained for the results presented in Fig. 10. The effect from the elliptical beams also applies to the experimental results. 

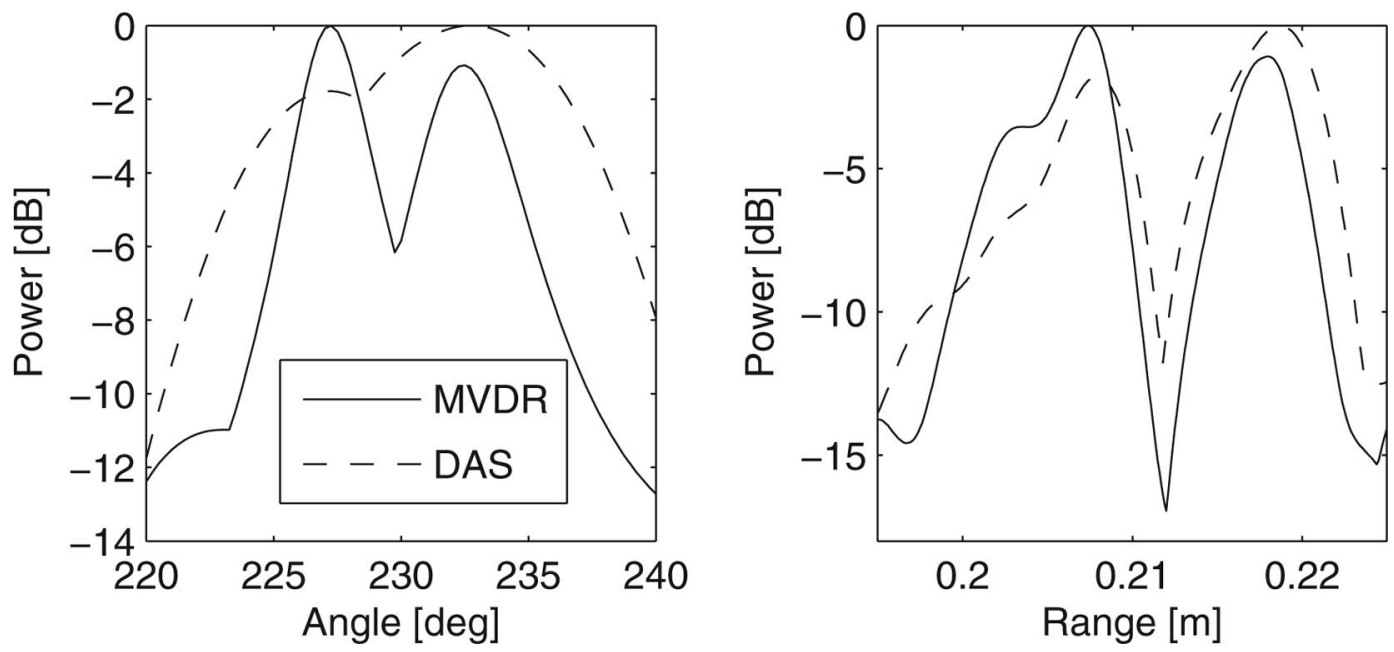

Fig. 11. Angle and range resolvability of hole pair A using the MVDR and DAS on measured data from Array 2 on the 6-mm plate.

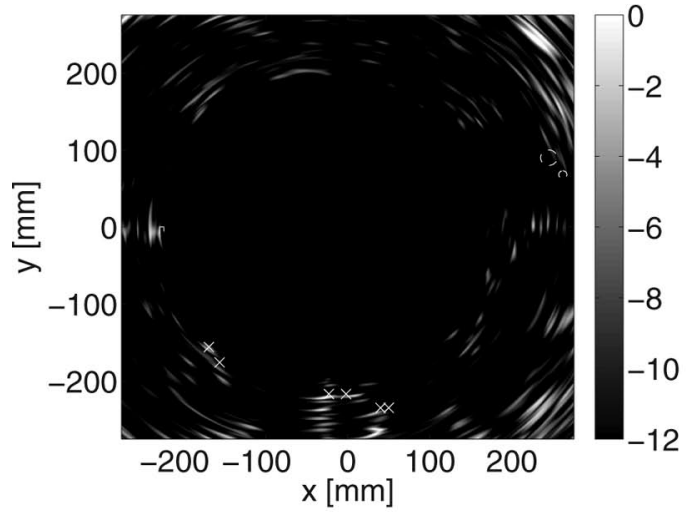

(a)

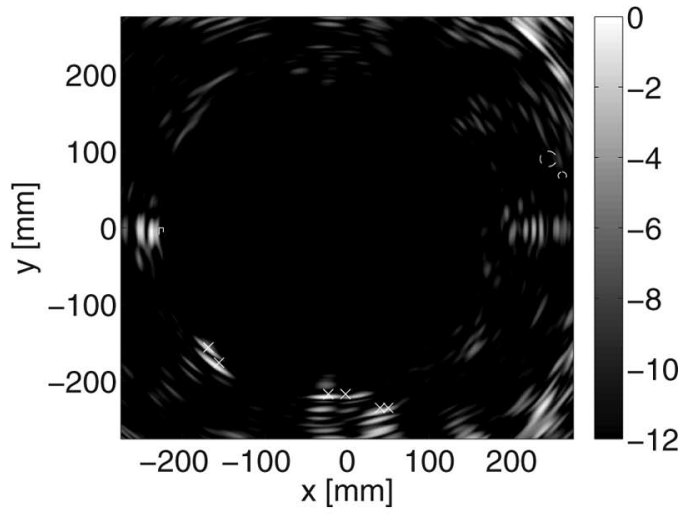

(b)

Fig. 12. The images resulting from the measured data using Array 2 with a $+3 \%$ change in the wave velocity. MVDR (a) and DAS (b). Cut at $-12 \mathrm{~dB}$

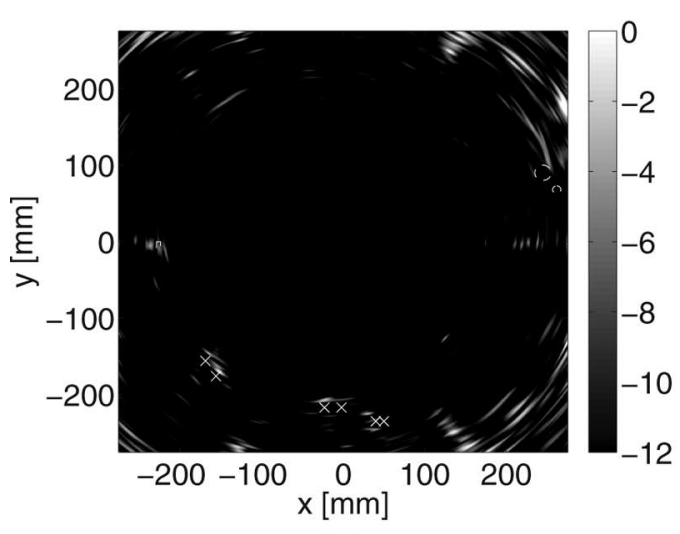

(a)

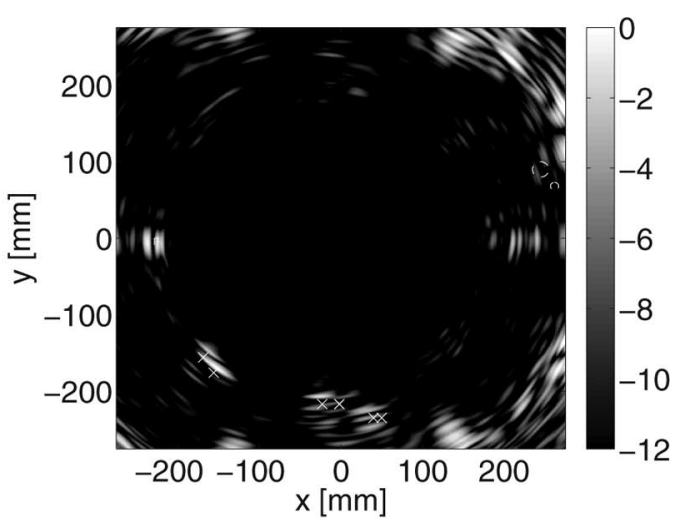

(b)

Fig. 13. The images resulting from the measured data using Array 2 with a $-3 \%$ change in the wave velocity. MVDR (a) and DAS (b). Cut at $-12 \mathrm{~dB}$.

To evaluate the sensitivity to uncertainties in the plate characteristics, dispersion curves were calculated based on a $\pm 3 \%$ change in the longitudinal and shear velocity using Array 2 with $7 \mathrm{~mm}$ element spacing (all other settings were identical). Fig. 12 presents the results analogous to those in Fig. 10 but for a $+3 \%$ change in the longitudinal and shear wave velocities, whereas Fig. 13 shows the corresponding result for a $-3 \%$ change.

\section{Discussion}

From both the simulated and experimental results presented in the previous sections it is apparent that the MVDR approach performs much better than the DAS 
method in terms of resolution, side lobe suppression, and interfering mode suppression. For some cases, the MVDR approach has problems with underestimation of the amplitudes. This may be caused by either steering vector errors, or signal cancellation caused by the correlated backscattered signals. This will be discussed in detail in this section.

From the profiles in Figs. 8 and 11, it is clear that the MVDR approach yields higher resolution than the DAS method on both simulated and experimental data. Underestimation is most clearly seen in the simulated results, in which the DAS correctly estimates the same amplitude for both defects, whereas the MVDR underestimates the amplitude of one of the holes.

Note also that the range resolution of the MVDR approach is better, which might not be expected. This is because the steering vector for near-field reflectors is rangedependent, and may therefore improve range resolution. Hence, because hole pair C is in the near-field of Array 2, the MVDR can perform better than the DAS approach. In the far-field, the range resolution will be equal for both approaches.

Even though the range and angle profiles are slightly distorted, it may be of interest to make a comparison with the theoretical far-field resolutions. The location of hole pair $\mathrm{C}$, used for the profiles, makes the effective aperture size a linear array with a length of approximately the diagonal of the array. The angular resolution of the DAS approach as the half-power beamwidth in degrees is $50.8 \lambda / D$, where $\lambda$ is the wavelength. This makes the expected farfield resolution of the DAS approach approximately $7.7^{\circ}$, which seems reasonable when observing Fig. 8. For a nondispersed pulse the range resolution is limited by $c /(2 B)$, where $c$ is the velocity and $B$ is the bandwidth. The group velocity of the $A_{0}$ mode is $3182 \mathrm{~m} / \mathrm{s}$ at $280 \mathrm{kHz}$, making the minimum resolvable distance between two reflectors approximately $6.1 \mathrm{~mm}$. The difference in range between hole pair $\mathrm{C}$ is $5.2 \mathrm{~mm}$. This is confirmed by observing that the peaks are not clearly resolved (only $-2 \mathrm{~dB}$ between the peaks) in the range plot of Fig. 8.

Recall that the focusing was performed between 50 and $450 \mathrm{kHz}$, which corresponds to wavenumbers between 200 and $1000 \mathrm{rad} / \mathrm{m}$ for the $A_{0}$ mode. The wider Array 2 yielded higher resolution but also proved to be more sensitive to the $S_{0}$ mode. To explain the reason for this increased sensitivity, Fig. 14 shows the steered response of Array 2 when steered to angle $0^{\circ}$ and wavenumber $600 \mathrm{rad} / \mathrm{m}$. The steered response shows the output from an unweighted steered beamformer for a range of wavenumbers. The figure illustrates aliasing for the URA, where a grating lobe can be seen at $300 \mathrm{rad} / \mathrm{m}$ on the opposite side $\left(180^{\circ}\right)$ of the array. When steering the beamformer over the $A_{0}$ mode's range of wavenumbers from $0^{\circ}$, the grating lobe will pass over the wavenumber range of the $S_{0}$ mode. The $S_{0}$ mode is faster and the edge-reflected $S_{0}$ mode will therefore appear to come from the inner area of the plate. Because of the grating lobe, the edge-reflected $S_{0}$ mode from the opposite side of the array will contribute to the edge-reflected $S_{0}$

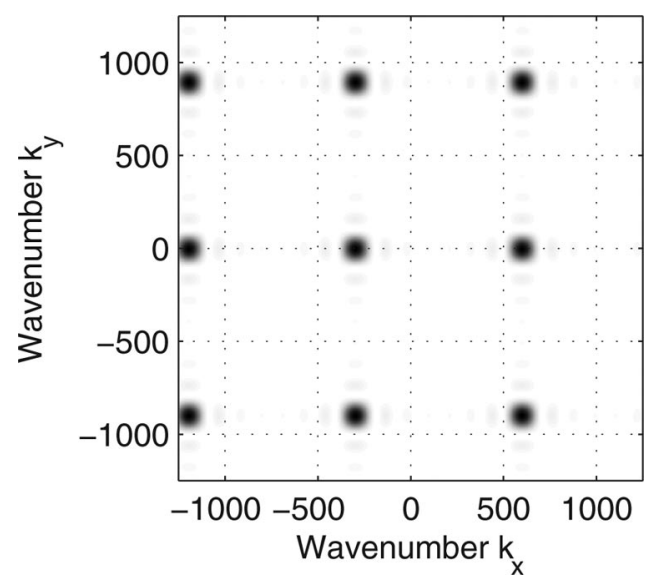

Fig. 14. Steered response of Array 2 when steered to angle $0^{\circ}$ and wavenumber $600 \mathrm{rad} / \mathrm{m}$. Note that $k_{x}$ and $k_{y}$ represent the direction of arrival, and not as in the typical response plots: the direction of propagation.

mode from $0^{\circ}$, which will lead to more significant interference. Array 1, having smaller element spacing, has twice the distance between the grating lobes and the results therefore show less interference from the $S_{0}$ mode.

The interference seen in the simulated results for Array 1 in Fig. 6 is mainly caused by the $S_{0}$ mode at $90^{\circ}$ intervals, with corner reflections in between. More severe interference appears for Array 2 in Fig. 7, where aliasing effects from both the $S_{0}$ and the $A_{0}$ mode can be seen in addition to the non-aliased $S_{0}$ mode. The interference is smeared out because the frequency components for the aliasing peaks are not coherently phase adjusted. From the results, it is apparent that the MVDR algorithm better suppresses aliasing from the $A_{0}$ and $S_{0}$ modes as well as the interference from the $S_{0}$ mode than the DAS. However, the highly effective suppression of the aliasing from the $A_{0}$ and $S_{0}$ modes is only possible because the aliasing occurs in the near-field. Far-field aliasing occurs because the steering vector is identical for two different incident angles and possibly wavenumbers, which makes it impossible for the MVDR algorithm to discriminate the two signals. In near-field, there is also a range component in the steering vector, which may lead to non-identical steering vectors because the range does not properly align the aliasing wavenumber from the other direction. This enables the MVDR approach to suppress the aliasing seen in the DAS results in Fig. 7.

The two major causes of underestimation are steering vector errors and adjacent defects. Steering vector errors, caused by, for instance, errors in the dispersion characteristics, can potentially cause the MVDR filter to underestimate amplitude. The effect of steering vector errors can be seen in Figs. 12 and 13, where the effects of errors in the dispersion characteristics are shown. The performance of the MVDR is much poorer in this situation, but still matches the performance of the DAS. The DAS shows similar behavior with and without steering vector errors, besides the obvious errors in position.

Signal cancellation caused by closely spaced defects is an important issue for the MVDR algorithm. In the simu- 
lation results using Array 2 in Fig. 7, the reflections from the pair of holes labeled $\mathrm{C}$ seem to cancel, which leads to underestimation of the amplitude. The cancellation was much less severe when using Array 1 even with less diagonal loading (Fig. 6). The problems were not apparent for the DAS approach for either of the arrays in the simulations. In the experimental results, the underestimation problem was most severe for Array 1 as can be seen from Fig. 9. Both methods yield different amplitudes for the holes A but show reasonable accuracy in position. The MVDR has significant problems with both power and position of pairs B and C. The DAS approach yields more reasonable amplitude estimates but it is very difficult to determine the positions of the defects.

In the experimental results for Array 2 presented in Fig. 10, both holes in pair B are detected. Although both methods estimate the amplitude of the right hole lower than for the left, its position is too far to the right. Pair $\mathrm{C}$ cannot be resolved and is detected as a single defect. Both methods underestimate the amplitude but the MVDR more severely. Because both methods have problems with pairs $\mathrm{B}$ and $\mathrm{C}$, it cannot be concluded that this is an effect related to the MVDR approach. Effects from steering vector errors can be reduced by using either a higher level of diagonal loading or by increasing the number of subarrays used for spatial smoothing, but this is achieved at the cost of lower resolution and reduced interference suppression.

The saturation of the edge reflections created a significant amount of noise in post-processed data from both algorithms in the areas closer than $100 \mathrm{~mm}$ to the edges, which is the reason for not showing images covering the whole plate. After increasing the amplitude range of the oscilloscope, and thereby avoiding saturation, these problems disappeared. Unfortunately, the insufficient dynamic range made the much weaker signals from the defects undetectable using either of the algorithms.

An obvious limitation of the measurement setup is the simultaneous use of only 8 array elements, which hindered assessing the potential effects of interelement scattering that may be encountered for a full 2-D array.

\section{Conclusions}

A method for adaptive beamforming of Lamb waves has been presented in the paper. The dispersion of the Lamb modes was compensated using theoretically predicted dispersion curves. Dispersion compensated data was processed using both a standard DAS beamformer and the MVDR beamformer. Simulated and experimental results show that the MVDR approach can yield better performance compared with the standard DAS approach in terms of higher resolution and better suppression of interfering Lamb modes.

Signal cancellation, causing underestimation of the signal amplitudes, is an issue that needs to be addressed when working with the MVDR algorithm. Increasing the number of subarrays used for spatial smoothing can reduce signal cancellation effects and increase robustness at the cost of lower resolution and adaptivity of the filter. Diagonal loading also reduces signal cancellation to some degree and improves robustness of the estimation.

The MVDR algorithm presented in the paper can be easily generalized to an active array with multiple transmitters.

\section{REFERENCES}

[1] P. D. Wilcox, M. J. S. Lowe, and P. Cawley, "Lamb and SH wave transducer arrays for the inspection of large areas of thick plates," in Annu. Rev. Progress in QNDE, vol. 19A, 2000, pp. 1049-1056.

[2] V. Giurgiutiu and J. Bao, "Embedded ultrasonic structural radar for the nondestructive evaluation of thin-wall structures," in Proc. 2002 ASME Int. Mechanical Engineering Congress, 2002, pp. 17-22.

[3] P. D. Wilcox, "Omni-directional guided wave transducer arrays for the rapid inspection of large areas of plate structures," IEEE Trans. Ultrason. Ferroelectr. Freq. Control, vol. 50, no. 6, pp. 699-709, 2003

[4] C. H. Wang, J. T. Rose, and F. K. Chang, "A synthetic time-reversal imaging method for structural health monitoring," Smart Mater. Struct., vol. 13, no. 2, pp. 415-423, 2004.

[5] F. Yan and J. L. Rose, "Guided wave phased array beam steering in composite plates," in Proc. SPIE, vol. 6532, 2007, art. no. 65320G.

[6] L. Yu, V. Giurgiutiu, and J. Kendall, "Omnidirectional guided wave PWAS phased array for thin-wall structure damage detection," in Proc. SPIE, vol. 6529, 2007, art. no. 652939.

[7] F. Yan, R. L. Royer Jr., and J. L. Rose, "Ultrasonic guided wave imaging techniques in structural health monitoring," J. Intell. Mater. Syst. Struct., vol. 21, no. 3, pp. 377-384, 2010.

[8] V. Giurgiutiu, "Tuned Lamb wave excitation and detection with piezoelectric wafer active sensors for structural health monitoring," J. Intell. Mater. Syst. Struct., vol. 16, no. 2, pp. 291-305, 2005.

[9] J. L. Rose, Ultrasonic Waves in Solid Media. New York, NY: Cambridge University Press, 1999.

[10] P. D. Wilcox, "A rapid signal processing technique to remove the effect of dispersion from guided wave signals," IEEE Trans. Ultrason. Ferroelectr. Freq. Control, vol. 50, no. 4, pp. 419-427, 2003.

[11] E. Moulin, N. Bourasseau, J. Assaad, and C. Delebarre, "Lamb wave beam-steering for integrated health monitoring applications," in Proc. SPIE, vol. 5046, 2003, pp. 124-131.

[12] P. Fromme, P. D. Wilcox, M. J. S. Lowe, and P. Cawley, "On the development and testing of a guided ultrasonic wave array for structural integrity monitoring," IEEE Trans. Ultrason. Ferroelectr. Freq. Control, vol. 53, no. 4, pp. 777-785, 2006.

[13] A. Velichko and P. D. Wilcox, "Guided wave arrays for high resolution inspection," J. Acoust. Soc. Am., vol. 123, no. 1, pp. 186-196, 2008.

[14] T. Stepinski and M. Engholm, "Piezoelectric circular array for structural health monitoring using plate waves," in 7th Int. Workshop on Structural Health Monitoring, Stanford, CA, Sep. 2009.

[15] M. Sasso and C. Cohen-Bacrie, "Medical ultrasound imaging using the fully adaptive beamformer," in IEEE Int. Conf. Acoustics, Speech and Signal Processing, vol. 2, 2005, pp. 489-492.

[16] J. F. Synnevåg, A. Austeng, and S. Holm, "Adaptive beamforming applied to medical ultrasound imaging," IEEE Trans. Ultrason. Ferroelectr. Freq. Control, vol. 54, no. 8, pp. 1606-1613, 2007.

[17] J. D. Achenbach and Y. Xu, "Wave motion in an isotropic elastic layer generated by a time-harmonic point load of arbitrary direction," J. Acoust. Soc. Am., vol. 106, no. 1, pp. 83-90, 1999.

[18] H. L. Van Trees, Optimum Array Processing (Detection, Estimation, and Modulation Theory, Part IV), 1st ed., New York, NY: WileyInterscience, 2002.

[19] J. Capon, "High-resolution frequency-wavenumber spectrum analysis," Proc. IEEE, vol. 57, no. 8, pp. 1408-1418, 1969.

[20] P. Stoica and R. L. Moses, Introduction to Spectral Analysis. Upper Saddle River, NJ: Prentice Hall, 1997.

[21] J. Krolik and D. Swingler, "Multiple broad-band source location using steered covariance matrices," IEEE Trans. Acoust. Speech Signal Process., vol. 37, no. 10, pp. 1481-1494, 1989.

[22] T. J. Shan, M. Wax, and T. Kailath, "On spatial smoothing for directon-of-arrival estimation of coherent signals," IEEE Trans. Acoust. Speech Signal Process., vol. 33, no. 4, pp. 806-811, 1985. 
[23] H. Hung and M. Kaveh, "Focussing matrices for coherent signalsubspace processing," IEEE Trans. Acoust. Speech Signal Process., vol. 36 , no. 8 , pp. $1272-1281,1988$.

[24] A. B. Gershman and V. T. Ermolaev, "Optimal subarray size for spatial smoothing," IEEE Signal Process. Lett., vol. 2, no. 2, pp. 28-30, 1995.

[25] J. Li, P. Stoica, and Z. Wang, "On robust capon beamforming and diagonal loading," IEEE Trans. Signal Process., vol. 51, no. 7, pp. 1702-1715, 2003.

[26] D. N. Alleyne and P. Cawley, "A two-dimensional fourier transform method for the measurement of propagating multimode signals," $J$. Acoust. Soc. Am., vol. 89, no. 3, pp. 1159-1168, 1991.

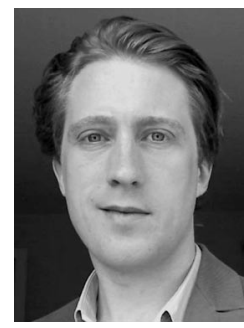

Marcus Engholm was born in Sundvall, Sweden, in 1977. He earned M.Sc. and Ph.D. degrees from Uppsala University in 2002 and 2010, respectively. His research interests include signal processing in non-destructive evaluation, ultrasonic resonance inspection techniques, and ultrasonic transducer modeling.

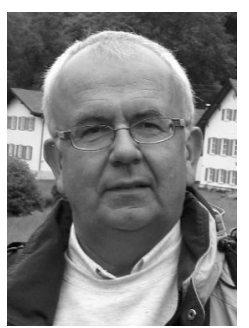

Tadeusz Stepinski was born in December 1950 in Poland. He obtained his M.Sc. degree in electrical engineering from the Technical University of Szczecin. Poland, in 1973 and the Ph.D. degree in technology from the Technical University of Warsaw, Poland, in 1983.

Since 1988, he has been with Signals and Systems at Uppsala University, Sweden. He initiated research activity in the field of nondestructive evaluation and structural health monitoring at Uppsala University. His research interests are in the areas of ultrasound, array processing, digital signal processing, and measurement engineering. In 2002, he was appointed full Professor in Electrical Measurement Engineering at Uppsala University. He is author and co-author of more than 120 papers.

Dr. Stepinski is a Senior Member of IEEE, and a member of the Acoustical Society of America, ASNT (American Society for NDT), and the British Institute of Non-Destructive Testing. He chairs the Committee for New Techniques and Development in FOP (the Swedish Society for NDE). 